\title{
LA POLÍTICA INTERNACIONAL SUBNACIONAL: DESAFÍOS PARA LOS MUNICIPIOS DE LA PROVINCIA DE BUENOS AIRES EN EL SIGLO XXI
}

\author{
Subnational international Policy: Challenges for the \\ MUNICIPALITIES OF THE PROVINCE OF BUENOS AIRES IN THE 21 \\ CENTURY
}

Juan Cruz Tisera

María Soledad Manassero

Ricardo Sebastián Piana

\begin{abstract}
RESUMEN
Dentro del regionalismo sudamericano y estableciendo los desafíos que nos imprime la actual Sociedad Internacional, ahora global, la Política Internacional Subnacional se presenta como un fenómeno relativamente novedoso en el contexto de la provincia de Buenos Aires. Buscamos identificar características del proceso de influencia de doble vinculación entre la sociedad internacional y los gobiernos subnacionales (municipales), describiendo un fenómeno que ha sido identificado como glocal. Desde el campo de las Relaciones Internacionales, proponemos establecer un marco teórico que analiza el despertar internacional de los actores subnacionales. En un contexto denominado Sociedad Internacional Global, se determinan las modificaciones del carácter central y unitario del Estado-Nación propuesto por el realismo político.
\end{abstract}

\section{PALABRAS CLAVE}

Teoría - Política Internacional Subnacional - Sociedad Internacional- Gobiernos Subnacionales - Interdependencia.

\section{ABSTRACT}

Within the South American regionalism and establishing the challenges that the present International Society, now global, gives us, Subnational International Politics is presented as a relatively new phenomenon in the context of the province of Buenos Aires. We seek to identify the characteristics of the process of influence of double bond between international society and subnational (municipal) governments, describing a phenomenon that has been identified as glocal. From the field of International Relations, we propose to establish a theoretical framework that analyzes the international awakening of subnational actors. In a context called the International Global Society, the modifications of the central and unitary nature of the NationState proposed by political realism are determined.

\section{KEY WORDS}

Theory - Subnational International Politics - International Society - Subnational Governments Interdependence. 


\section{INTRODUCCIÓN}

Nos encontramos en una sociedad internacional de carácter global, o como algunos teóricos sostienen, una era postwestfaliana muy diferente a la existente hasta entonces dado el debilitamiento de los principales elementos que se afirmaron a partir de 1648 y que marcaron la sociedad internacional hasta la segunda mitad del siglo XX (Buzan y Little, 2000, pp. 2-6). Las últimas décadas han traído consigo una dinámica de cambios profundos a partir del desarrollo de la globalización, integrando una profunda transformación en la Sociedad Internacional. Como sostuvo Truyol, estamos frente a una "verdadera mutación de la sociedad internacional" (1993, p. 26), caracterizada por una dimensión de la Globalización, Interdependencia Compleja y Mundialización, determinado por la dialéctica global-glocal. Es en este contexto que los actores subnacionales, como otro de los nuevos actores internacionales, buscan generar políticas públicas y capacidades estatales que les permitan potenciar las acciones en el exterior.

Creemos necesario hablar de una Sociedad Internacional Global reconociendo la existencia de otros términos como Escenario Internacional; Sistema Internacional; Vida Internacional, entre otros tantos compartidos por la academia. En nuestra opinión, el término adecuado es el de Sociedad Internacional Global y por ello hacemos referencia a la existencia de tres realidades sociales que coexisten e interactúan: el sistema político-diplomático, el sistema transnacional y la sociedad humana (Arenal, 2005, pp. 453-464). Según Hedley Bull (1977) es necesario hacer una distinción entre Sistema Internacional y Sociedad Internacional, al primero lo define como dos o más Estados que tienen contacto suficiente entre ellos, mientras que la Sociedad Internacional "existe cuando un grupo de Estados, concientes de determinados intereses y valores comunes, forman una sociedad en el sentido de que se conciben a sí mismos como sujetos a un conjunto de normas en su relación con los otros, y participan en el funcionamiento de las instituciones comunes. Según los planteos del autor, la Sociedad Internacional tiene cuatro objetivos universales de vida: la preservación del sistema y la sociedad en sí mismo; el mantenimiento y la independencia de la soberanía de los Estados (sobre todo la externa); el 
mantenimiento de la paz como ausencia de guerra entre los Estados ${ }^{1}$; y por último, la estabilidad de las estructuras que posibilitan cumplir la palabra y los contratos, limitando de este modo la violencia.

Hoffmann (1990, pp. 115-122) y Nye (2003, pp. 66-67) sostienen que lo que históricamente ha variado de una sociedad internacional a otra ha sido la importancia y el protagonismo que cada una de esas realidades sociales han tenido en las sociedades internacionales del momento y las interacciones que se han establecido entre ellas.

Con todo, de las tres realidades sociales que conforman la sociedad internacional es, lógicamente, el sistema político-diplomático, en cuanto expresión del fenómeno de descentralización del poder, el que proporciona al menos formalmente los rasgos definitorios de cada sociedad internacional y el que genera normalmente la existencia de normas e instituciones comunes que regulan el funcionamiento de esa sociedad internacional, especialmente a nivel del propio sistema político-diplomático. Todo ello supone que de las tres realidades sociales que configuran la sociedad internacional es el sistema político-diplomático la única que tiene un relativo grado de institucionalización y formalización, que varía notablemente, como es lógico, en función de los distintos tipos de sociedad internacional que han existido históricamente (Arenal, 2005, pp. 460-461).

Dentro del regionalismo sudamericano y estableciendo los desafíos que nos imprime la actual Sociedad Internacional, ahora global, la Política Internacional Subnacional es un fenómeno relativamente novedoso (en lo teórico pero no en las prácticas), y que se encuentra en constante transformación, un proceso que ha comenzado a generar conceptos e interpretaciones que no siempre conducen a una mejor comprensión del mismo (Calvento, 2015). Para no entrar en conclusiones erróneas, creemos necesario profundizar el debate teórico y ampliar los estudios empíricos, generando nuevos interrogantes que permitan comprender el proceso actual.

\footnotetext{
${ }^{1}$ Este objetivo se diferencia de Bobbio quien sitúa a la paz en primer término.
} 
Entendemos necesario estudiar las realidades de los municipios siguiendo a Beck, cuando afirma que "Lo global (lo grande) sólo puede ser comprendido en función de lo concreto, lo pequeño, lo local" (Beck, 2008, pp. 76-77). En efecto, mientras los actores tradicionales de las relaciones internacionales se encuentran encorsetados en complejas relaciones y condicionamientos políticos y económicos, los actores subnacionales han demostrado mayor flexibilidad e innovación.

De esta manera buscamos considerar a los actores subnacionales que se interrelacionan internacionalemente, en nuestro caso los municipios. Definimos a los actores subnacionales como "las unidades institucionales, o niveles del poder ejecutivo, que son componentes de un gobierno de un Estado Nacional: estados, regiones, provincias, municipios u otros poderes locales" (Fronzaglia, 2005, p. 44).

Centrando el desarrollo en relación con el lema "Pensar Globalmente y Actuar Localmente" (Cruz y Bodnar, 2008, pp. 13-25), buscamos contribuir en la construcción de un marco teórico desde las relaciones internacionales, identificando el accionar internacional de los actores subnacionales, en particular los municipios, con el objetivo de generar, a futuro, modelos explicativos que nos permitan comprender su accionar, señalando sus herramientas de relacionamiento y posicionamiento en el actual escenario internacional-global.

En el contexto de lo glocal este artículo pretende profundizar los principales enfoques y herramientas desde el campo de las relaciones internacionales, conceptualizando la inserción internacional de los actores subnacionales en la Provincia de Buenos Aires y describir las posibilidades que tienen los municipios de la Provincia de Buenos Aires, en un contexto de limitaciones normativas pero amplios canales de vinculación ya consolidados con otros actores de una sociedad internacional global.

En la primera parte del artículo, nos proponemos estudiar la creciente interdependencia como una de las dinámicas de la actual Sociedad Internacional Global. La interdependencia se desprende de la conceptualización de la globalización y comienza a adquirir características autónomas, hecho relevante para comprender las profundas transformaciones que experimenta la actual realidad internacional. Desde 
esta perspectiva ubicamos la aparición de los actores de carácter subnacional, identificados en los municipios de la Provincia de Buenos Aires.

Seguidamente, nos detendremos en el concepto de política internacional subnacional, identificando las diversas formas de posicionamiento y relacionamientos de los municipios en el exterior.

Finalmente, estudiamos las experiencias y posibilidades de los municipios de la Provincia de Buenos Aires, en el contexto de las restricciones normativas que aún existen en Argentina, tanto desde la normativa nacional como provincial.

\section{LA CRECIENTE INTERDEPENDENCIA}

Como sostuvieron R. Keohane y J. Nye, "vivimos en una era de interdependencia. Esta vaga afirmación expresa pobremente un sobreentendido que, sin embargo, corresponde a un difundido sentimiento de que la propia naturaleza de la política mundial está cambiando" (1988, p. 19). El enfoque conocido como interdependencia compleja tuvo su desarrollo a partir de las propuestas trabajadas por Keohane y Nye, dentro del seno del realismo político estadounidense y que posteriormente logra alcanzar un análisis más profundo sosteniendo que "en lenguaje común, dependencia significa un estado en que se es determinado o significativamente afectado por fuerzas externas. Interdependencia, en su definición más simple, significa dependencia mutua. En política mundial, interdependencia se refiere a situaciones caracterizadas por efectos recíprocos entre países o entre actores en diferentes países" (1988, p. 22). La interdependencia incorpora una pluralidad de actores de características no estatales: organismos internacionales, ONG, empresas multinacionales, individuos, y por último, el rol de las ciudades globales en el proceso conocido como glocalización. De esta manera el Estado pierde el rasgo distintivo que pregonaban los tradicionalistas, entrando en competencia con otros actores. Lo local rompe con la lógica clásica de los Estados, generando múltiples conexiones (Piana y Tisera, 2017).

En igual sentido se ha dicho que "Dado que la creciente interdependencia es hoy parte integral de la globalización, sus efectos no pueden separarse de los que se derivan de esta última" (Arenal, 2009, p. 211). 
Proponemos destacar tres características esenciales que nos permitirán entender su análisis: ${ }^{2}$

1. Canales Múltiples: canales que conectan de diversas formas a las sociedades, que pueden darse de manera formal o informal. Los realistas sostenían que sólo se producían relaciones entre Estados; este enfoque señala que además hay relaciones transgubernamentales, se producen relaciones transnacionales. De esta manera las políticas locales cobran una relevancia que en el juego del pasado dependía exclusivamente de los Estados y sus intereses nacionales a nivel global.

2. Ausencia de jerarquías: la agenda de los Estados estará compuesta por una variedad de temas -más allá del militar- y se caracterizará por no poseer una jerarquía entre los asuntos que componen dicha agenda: así la seguridad dejará de dominar el establecimiento de las cuestiones a tratar. Este punto es altamente demostrable a partir de la década de los setenta y la incorporación de una variedad de temas ajenos a los militares. Múltiples temas pueden afectar a un determinado sector o grupo, aunque no afecte a toda la nación. De esta manera se hace más compleja la formulación de una política pública, que ahora estará dominada por varios temas.

3. Menor papel de las fuerzas armadas: uno de los preceptos básicos de este enfoque sostiene que en un mundo caracterizado por la interdependencia compleja, los Estados no utilizan la fuerza militar contra otros Estados. Esto es cierto cuando analizamos la imposibilidad de resolver un conflicto económico entre Estados de una misma región. Una de las premisas centrales del realismo era que la fuerza militar subordinaba otros medios de poder, la distención, la disminución al temor de un ataque por parte de otro Estado, los costos elevados de una invasión, o la imposibilidad de solucionar a través de la fuerza problemas tales como la degradación del medioambiente han dejado lugar a la aparición de otros instrumentos de poder, más allá del militar.

2 Para un análisis mayor sobre las características de la interdependencia compleja ver (Keohane y Nye, 1988, pp. 41-47). 
A partir de este primer análisis surgirán tres premisas que darán origen a lo que Keohane y Nye denominan como los procesos políticos de la interdependencia compleja: ${ }^{3}$

1. Estrategias de vinculación: el análisis clásico decía que los Estados que tenían el poder militar dominaban el sistema internacional; en un mundo caracterizado por la interdependencia, la fuerza militar se encuentra desvalorizada. De esta manera los Estados fuertes se vincularán a través de la fuerza y los Estados más débiles encontrarán su vinculación generalmente en los organismos internacionales. La disminución del poder militar lleva a que los Estados generan otras estrategias de vinculación. En las últimas décadas lo local ha comenzado a ser el centro de las estrategias de vinculación entre los Estados, ciudades globales que piensan globalmente y actúan localmente.

2. Establecimiento de la agenda: la falta de una jerarquía de temas determina relaciones más complejas; las cuestiones militares seguirán en la agenda, pero en un mundo de interdependencia compleja ciertos temas adquirirán una relevancia preponderante: la política monetaria, cuestiones de medioambiente, los flujos de personas, la influencia de las empresas multinacionales, la articulación de demandas de la sociedad civil y el rol de las ciudades globales se constituyen en temáticas importantes en la agenda.

3. Relaciones transnacionales y transgubernamentales: las dos características señaladas anteriormente hacen cada vez más difícil una distinción clara entre lo doméstico y lo internacional. La multiplicidad de actores y socios hace más compleja esta relación estableciéndose vinculaciones entre Estados, ciudades, y actores no estatales.

La interdependencia afecta la política mundial y el comportamiento de los Estados pero las acciones gubernamentales también influyen sobre los modelos de interdependencia: al aceptar procedimientos, normas o instituciones para ciertas clases de actividades, los gobiernos regulan y controlan las relaciones transnacionales e interestatales.

3 Para un análisis mayor sobre las características de los procesos políticos de la interdependencia compleja ver (Keohane y Nye, 1988, pp. 47-56). 
La interdependencia compleja nos permite establecer patrones de conducta diferentes a la concepción realista; si bien es conveniente destacar que no se alejan por completo de sus desarrollos, podemos señalarla como el primer cuestionamiento serio que se les hacen a los teóricos clásicos.

En un mundo complejo este enfoque nos permite realizar una mejor aproximación de nuestro estudio porque comprendemos la interdependencia no sólo entre Estados sino entre estos, las sociedades civiles, las organizaciones no gubernamentales, las empresas y las ciudades ${ }^{4}$. Como sostuvo Nye, los procesos de transformación que genera la interdependencia compleja en la Sociedad Internacional, tanto en lo económico, político, sociocultural y científico-tecnológico "elevaron la importancia de los asuntos transnacionales y facultaron a diferentes actores para tener un papel más amplio en la política mundial" (2003, p. 2). Por esto es que identificamos la participación ineludible de una variedad de actores además de los Estados Nacionales: un escenario que se visualiza como favorable para la participación de actores subnacionales, que no son considerados como sujetos del derecho internacional clásico, pero que intervienen en diversas modalidades de acuerdos, entendimientos y hermanamientos de cooperación internacional de manera formal o informal y desde ámbitos económicos y/o culturales.

\section{Pensar globalmente y Actuar localmente. la política internacional SUBNACIONAL}

Definimos a la Política Internacional Subnacional como "la decisión política y la herramienta pública de los gobiernos locales que se ocupa de impulsar la inserción internacional, a través de una estrategia y objetivos tendientes a aprovechar, articuladamente, las oportunidades del contexto exterior con las necesidades del territorio" (Calvento, 2016, p. 309). De esta manera buscamos considerar a los actores que integran un Estado nacional y por ello identificamos a los actores subnacionales como estados, regiones, provincias o municipios, que son reconocidos por los Estados

\footnotetext{
${ }^{4}$ En ese sentido Arenal señala: "El constante incremento de los niveles de interdependencia ha transformado radicalmente la sociedad internacional y de manera muy especial el comportamiento de los Estados, contribuyendo de manera decisiva a conformar una sociedad internacional postwestfaliana, como consecuencia de los decisivos efectos que se han derivado de esta dinámica" (Arenal, 2009, p. 209).
} 
Nacionales, forman parte, al interior de esta macro estructura jurídico política pero no se confunde con este por tener funciones y competencias propias o concurrentes.

Siguiendo la noción que nos propone Zubelzú (2008, p. 36), el empleo del término subnacional es el más difundido y empleado en las investigaciones que el de subestatal, aunque señala que "tanto el 'actor subnacional' como el 'actor subestatal' derivan de un mismo concepto integral: el 'Estado-Nación' [por lo cual] su uso resulta equivalente e indistinto".

Las últimas décadas del siglo $X X$ han traído consigo una dinámica de cambios profundos a partir del desarrollo de la globalización, integrando una profunda transformación en la Sociedad Internacional. Como sostuvo Truyol, estamos frente a una "verdadera mutación de la sociedad internacional" (1993, p. 26), caracterizada por una dimensión de la Globalización, Interdependencia Compleja y Mundialización, determinado por la dialéctica global-local. Es en este contexto que los actores subnacionales buscan generar políticas públicas que permitan potenciar las acciones en el exterior.

La modalidad de internacionalización que ejercen los actores subnacionales se determinan a partir del uso de la paradiplomacia, nueva diplomacia regional o protodiplomacia. Keating, define a la nueva diplomacia como un "tipo de actividad, que funciona por debajo o dentro de los intersticios del sistema tradicional de las relaciones internacionales" (2001, p. 2). El concepto paradiplomacia es definido por Zeraoui para "designar a las actividades internacionales de las instituciones no centrales de un país" (2007, p. 1). Según Meirelles Ribeiro (2008) la protodiplomacia se determina como una situación particular cuando un actor subnacional lleva a cabo acciones con el objeto de obtener mayor soberanía. Otro grupo de definiciones, señala que la paradiplomacia refiere a "Canales informales de participación que actúan de forma paralela a la diplomacia del Estado nacional, lo que denominan algunos autores como "paradiplomacia", que tiene que ver con las acciones en el exterior de las unidades territoriales subnacionales o gobiernos no centrales” (Ruiz Alanís, 2009, p. 256). 
Dentro de las herramientas y el accionar disponibles que presenta la política internacional subnacional pretendemos analizar el abanico disponible siempre teniendo en cuenta que "la participación externa de las entidades subnacionales es una política pública local, que materializa y aplica la estrategia de un gobierno de este nivel en el campo de las relaciones internacionales" (Calvento, 2016, p. 309).

El escenario internacional al que denominamos Sociedad Internacional Global, presenta una nueva dialéctica sobre la cuestión global-local. Por lo tanto, debemos considerar las "transformaciones globales" ${ }^{5}$ sobre este contexto, en un proceso que implica según Beck "un cambio de paradigma científico y un cambio de paradigma político" (2008, p. 129).

La visión realista de las relaciones internacionales y la hipótesis del Estado como único actor del sistema internacional, ya no es un modelo teórico válido. Un mundo interdependiente supone dos sucesos: en primer lugar, las reglas y por sobre todo, los conceptos centrales de esa visión teórica pierden su función explicativa: definiciones como "Estado Nacional", "Capitalismo Nacional", "Soberanía Nacional", entre otros, pierden su utilidad porque referencian situaciones y valores que ya no describen la compleja realidad del sistema internacional y, por ende, no pueden explicarlo. En segundo lugar, el proceso globalizador permite generar nuevos espacios y, por lo tanto, genera nuevos conceptos adaptados a una nueva realidad. De esta manera "la política se deslimita y desestataliza" (Beck, 2008, p. 27). En este proceso aparecen nuevos actores, con nuevos papeles, con reglas diferentes, generando la necesidad de identificar conceptos que se adaptan a una sociedad en cambio.

Ante las limitaciones estatales, se ha hecho cada vez más notorio la posibilidad de una relación en el desarrollo local y las dinámicas propuestas desde el ámbito global. El gobierno local implica el instrumento que impulsa la actividad internacional:

Por un lado, en clave económica, para la creación de lazos internacionales tendientes a estimular varias cuestiones que potencien y refuercen el desarrollo local, entre otras, el comercio, la inversión y el turismo. Por otro lado, en clave

\footnotetext{
${ }^{5}$ El concepto transformaciones globales es trabajado en extenso por los académicos Held, D., McGrew, A., Goldblatt, D. y Perration, J., (2002).
} 
política, el municipio es una herramienta fundamental para sostener la relación entre el desarrollo local y la dimensión externa, a través del diseño de un esquema de influencia institucional sobre las estructuras del gobierno central (Miranda, 2005, p. 7).

Como ha recordado Ruiz Alanís, ya en 1965 las Naciones Unidas, a través de la Resolución 2058 de su Asamblea General, estimaron que "el hermanamiento de ciudades representa uno de los medios de cooperación que deben fomentar las Naciones Unidas"6. Más tarde, en 1971, por Resolución 2861, estimó a la "cooperación intermunicipal mundial" como un complemento natural de la cooperación de los Estados y de las organizaciones intergubernamentales ${ }^{7}$.

"Pensar globalmente, actuar localmente", señala una apertura de lo particular a lo universal y este es justamente el desafío que se presenta a los actores subnacionales en la actual sociedad internacional. En este artículo entendemos a los municipios como los actores centrales ya que, como sostuvo Di Pietro "lo local, es la entidad subnacional de análisis, planificación y acción para el desarrollo que implica una serie de relaciones, comportamientos, pautas y convenciones comunes" (2001, p. 23). Sostenemos que lo local es: territorial, porque se despliega en un espacio determinado (municipal o regional); multidimensional, abarcando las distintas facetas de la vida humana; sistémico, porque supone la cooperación de los diversos actores de una comunidad; integrado, donde se articulan políticas públicas locales (presupuestos participativos); institucionalizado, se establecen reglas de juegos que regulan las conductas locales; e innovador, porque es el primer ámbito de exploración para los cambios.

Dentro de las herramientas y el accionar disponibles que presenta la política internacional subnacional pretendemos referenciar brevemente el abanico disponible siempre teniendo en cuenta que "la participación externa de las entidades subnacionales es una política pública local, que materializa y aplica la estrategia de un

\footnotetext{
${ }^{6}$ https://undocs.org/es/A/RES/2058(XX). Fecha de acceso 14/11/18.

${ }^{7}$ https://undocs.org/es/A/RES/2861(XXVI) Fecha de acceso 14/11/18.
} 
gobierno de este nivel en el campo de las relaciones internacionales" (Calvento, 2016, p. 309).

Mariana Calvento (2016), realiza una conceptualización que seguiremos en nuestro desarrollo operacionalizando y sistematizando la política internacional subnacional en dos dimensiones: de relacionamiento institucional internacional y de posicionamiento internacional.

En relación a las herramientas de relacionamiento institucional internacional, se destacan los acuerdos bilaterales ${ }^{8}$ donde cobran un rol protagónico los hermanamientos de ciudades $^{9}$, y en menor medida, acuerdos y convenios de cooperación internacional bilateral. En cuanto al posicionamiento internacional, los municipios buscan generar acuerdos de carácter multilateral con el objetivo de lograr posicionamiento exterior ${ }^{10}$. En una variedad de herramientas destacamos el desarrollo de políticas locales de comercio exterior cuyo objetivo prioritario se establece en generar condiciones favorables para el desarrollo de economías locales y regionales.

En esta línea, y de conformidad con el auge en la participación internacional de los actores subnacionales, ¿dónde y cómo se desarrolla la política internacional subnacional de los municipios de la Provincia de Buenos Aires?

\section{LAS RELACIONES INTERnACIONALES DE LOS MUNiCIPIOS DE LA PROVINCIA DE Buenos} AIRES

La posibilidad del relacionamiento internacional de las entidades subnacionales en Argentina está enmarcada en el artículo 124 de la Constitución Nacional, reformada en 1994, que señala que las Provincias pueden "celebrar convenios internacionales en tanto no sean incompatibles con la política exterior de la Nación y no afecten las

\footnotetext{
${ }^{8}$ Los acuerdos bilaterales "representan instrumentos concretos, tangibles y factibles de cooperación, asentados en una base común de voluntades e intereses a corto, mediano y largo plazo" (Batista, Jakobsen y Evangelista, 2008, p. 59).

${ }^{9}$ La importancia de este tipo de acciones se encuentra determinada a partir de una búsqueda que extienda las fronteras locales promoviendo el contacto entre culturas y pueblos diferentes.

10 "A diferencia de las políticas de relacionamiento institucional, son herramientas que dependen exclusivamente de la decisión y el trabajo unilateral de este actor" (Calvento, 2012, p. 321).
} 
facultades delegadas al Gobierno Federal o el crédito público de la Nación; con conocimiento del Congreso Nacional".

Si bien los entes autónomos no pueden técnicamente celebrar tratados o tener una política exterior ya que es una facultad exclusiva y excluyente del gobierno nacional, como en casi todos los Estados, la habilitación a las provincias se da en los términos de "convenios" para sus materias reservadas o concurrentes. Las provincias no pueden contradecir la política exterior del Estado ni su crédito para lo cual el conocimiento del Congreso es visto como un ámbito de concertación y colaboración ya que tampoco se le ha atribuido la facultad de aprobarlos o desecharlos como en el caso de los Estados Unidos de Norteamérica (Gelli, 2006, p. 1024).

En cuanto al desarrollo de esta facultad, la Constitución de la Provincia de Buenos Aires no tiene ningún tratamiento. Sin embargo, y tal como ha sistematizado el Consejo Argentino para las Relaciones Internacionales hasta 2015, la provincia tiene un variado y profuso listado de Convenios, Cartas, Entendimientos y Protocolos suscriptos con Estados nacionales pero espacialmente con otros actores subnacionales en el contexto de la creación de áreas institucionales específicas ${ }^{11}$.

Sin perjuicio de la autonomía de los municipios reconocida en la constitución nacional pero limitada aún por la legislación bonaerense, tampoco existen normativas específicas en la Ley Orgánica de Municipalidades, Decreto-Ley $N^{\circ} 6769 / 58$ y sus modificatorias $^{12}$. No obstante ello, los municipios desarrollan múltiples actividades que van desde acuerdos bilaterales, hermanamientos de ciudades, proyectos de cooperación técnica y financiera, realización de intercambios culturales, deportivos,

\footnotetext{
${ }^{11}$ Como recuerda Calvento (2010, p. 2) "en el caso de la Provincia de Buenos Aires, es preciso destacar que desde los años noventa, en consonancia con las nuevas tendencias nacionales, se crearon las primeras áreas específicas de gestión externa: la Subsecretaría de Relaciones Económicas Internacionales, principal agencia de gestión exterior de la Provincia, y la Dirección de Integración Regional, dependientes del Ministerio de Producción".

${ }^{12}$ De todas formas, la Ley $\mathrm{N}^{\circ} 24.807$ que crea la Federación Argentina de Municipios como entidad pública no estatal, prevé y le reconoce entre una de sus principales finalidades, objetivos y acciones "Establecer nexos y acuerdos con asociaciones afines de otros países, como también con organizaciones regionales e internacionales, con la finalidad de fomentar el intercambio de información, documentación y experiencias afines y alentar el desarrollo de programas y acciones de interés común" (art. $8^{\circ}$ inc. i).
} 
turísticos y de buenas prácticas en la gestión pública, participación y organización de ferias y congresos internacionales, entre otras actividades.

Ha señalado Perotti que:

La creciente actividad externa que han venido desarrollando las unidades subnacionales en la Argentina ha tenido causas de orden doméstico (como el regreso de la democracia, en la década de 1980, que actuó como un proceso movilizador de la participación e iniciativa de un amplio espectro de actores al interior del Estado y de la sociedad argentina, y posteriormente, el proceso de apertura de la economía en los años 90 del siglo $\mathrm{XX}$ ) y otras de carácter externo (como el propio proceso de globalización y el regionalismo) que han incidido fuertemente en la asunción por parte de las entidades subnacionales de actividades que, en otras épocas, pertenecían al ámbito de acción exclusivo del gobierno nacional (Perotti, 2010, p. 6).

En efecto, los municipios argentinos y en especial los bonaerenses desde los $90^{13}$, han desarrollado diversas estrategias de cooperación internacional descentralizada o paradiplomacia superando la limitación normativa.

En materia de relacionamiento institucional internacional, a partir de los datos recolectados, el hermanamiento de ciudades es la práctica más utilizada por los municipios, lo que requiere de la aprobación del ejecutivo y del legislativo local, como así también, del conocimiento de la Cancillería. A nivel general, se reconocen más de 400 experiencias entre gobiernos provinciales y municipales argentinos con actores extranjeros siendo, más del $80 \%$ de esos vínculos, los iniciados por los municipios, especialmente, de la Provincia de Buenos Aires ${ }^{14}$.

Otra de las vinculaciones de los municipios con el exterior surgen las relaciones multilaterales municipales, destacándose la creación de redes de ciudades que permiten una mayor generación de políticas de promoción e imagen de las ciudades.

\footnotetext{
${ }^{13}$ Sin embargo, desde el CIPPEC (2004), se señala que el crecimiento de esta política es a inicios de 2000.

${ }^{14}$ En 1967 el año se concretó el primer hermanamiento del cual se tiene su registro formal, entre el municipio de Bahía Blanca y la ciudad de Jacksonville, Estados Unidos.
} 
En nuestra región la red principal de municipios es la Red de Mercociudades ${ }^{15}$ constituyéndose como la mayor red regional de participación de estrategias de relacionamiento institucional regional. Lo distintivo de estas herramientas es que se establecen a partir de relaciones horizontales, que se realizan entre actores similares, con un gran margen de flexibilidad (Zapata Garesché, 2007). En la actualidad nos encontramos con la participación de más de ciento treinta municipios argentinos; configuran el $34 \%$ de los municipios que participan de esta política y son los que tienen más presencia en el grupo, aún por sobre Brasil. Entre los municipios bonaerenses destacan los de Avellaneda, La Plata, Mar del Plata, Bahía Blanca, Pilar, Vicente López, entre otros. Este tipo de estrategia ha permitido la construcción de una red horizontal de ciudades con un claro sentido de cooperación e intercambio entre sus socios a partir del trabajo de sus Unidades Temáticas ${ }^{16}$ (Chasquetti, 2006).

Otras de las estrategias de posicionamiento es la conocida como marketing internacional, la cual busca señalar las ventajas comparativas de una localidad, identificada en torno a la idea de Marca-Ciudad, la que pondera los atributos y las diferencias que identifican a una ciudad de otras. Dentro de estas estrategias podemos resaltar el lanzamiento en el año 2012 de marca "Ciudad de Avellaneda" identificando los productos y servicios necesarios para el desarrollo y la identificación del municipio en el exterior. Otro de los casos detectados es el desarrollo de la Marca -Ciudad en el Municipio de Morón hace ya más de una década, generando la construcción de slogan que identifica a los habitantes con la ciudad donde viven. Estas políticas permiten posicionar al municipio de manera competitiva ayudando a generar un posicionamiento en la memoria colectiva.

Con estas acciones identificamos la búsqueda de inserción internacional a partir de políticas de promoción y de imagen de los municipios. Ahora bien, también existen evidencias de posicionamiento internacional de los municipios de la Provincia de Buenos Aires. Algunos de los municipios que realizan actividades de comercio exterior son Quilmes, Mar del Plata, Tandil, Bahía Blanca, entre otros. En los últimos años el

\footnotetext{
${ }^{15} \mathrm{http}: / /$ www.mercociudades.org/

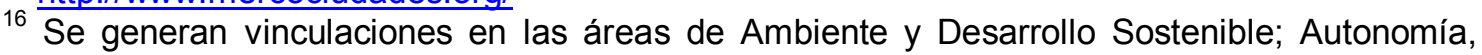
Gestión y Financiamiento Municipal; Cultura; Desarrollo Económico Local; Desarrollo Social; Desarrollo Urbano; Educación; integración Fronteriza; Turismo, entre otros.
} 
municipio de Lanús ha realizado una serie de actividades tendientes a favorecer el desarrollo local, como la "Casa de Lanús en el exterior", en articulación con el Banco de la Provincia de Buenos Aires, consiste en impulsar los negocios de las empresas locales, participando de las rondas de la Fundación Export-Ar. Otro ejemplo de este tipo de accionar se identifica en el Club de exportadores, un espacio cuyo objetivo es ser portavoz de las empresas locales, ofreciendo apoyo en la actividad internacional.

Finalmente, debemos destacar la faz institucional de estas políticas ${ }^{17}$. Ya son varios los municipios bonaerenses que, desde hace años, cuentan o han contado con oficias especializadas para trabajar estos acuerdos y vinculaciones. En efecto, podemos mencionar la oficina de Coordinación de Cooperación Internacional (hoy Dirección de Coordinación de la Gestión) de la ciudad de Pergamino, que impulsa el establecimiento y desarrollo de las relaciones interinstitucionales con el exterior, trabajando de manera transversal y articulada con otras áreas de gobierno. Junín, otra ciudad del interior de la provincia, cuenta con una Dirección General de Relaciones Internacionales que realiza las acciones vinculadas al Comercio Exterior, a las Relaciones Internacionales e Institucionales del Gobierno Local y la coordinación de Programas de Cooperación Internacional. Avellaneda cuenta con una Coordinación de Proyectos y Cooperación Internacional, mientras que en Quilmes, se desarrollan dentro de la Dirección General de Relaciones Regionales, Intermunicipales e Institucionales.

Algunas áreas específicas son temáticamente vinculadas a los aspectos comerciales, como la Dirección de Producción y Comercio Exterior en el Municipio de Vicente López o el de Morón, ya que dentro de la Secretaría de Producción y Desarrollo Económico, se tratan los aspectos vinculados a la promoción Económica y Comercio Internacional. En el caso de Bahía Blanca, "Bahía Internacional" tiene un perfil claramente orientado a la cooperación descentralizada. En otros casos, las acciones internacionales forman parte de la competencia de diversas áreas, como la Subsecretaria de Coordinación y Gestión de Proyectos en el caso del Municipio de Tandil.

\footnotetext{
${ }^{17}$ Tomamos esta dimensión porque se ha señalado su alto valor para la proyección de este tipo de políticas. En efecto, como señala Pallero (2014, p. 22) "La creación de la Oficina de Cooperación Internacional y Relaciones Internacionales tiene un impacto positivo para el gobierno local, se ha demostrado en otros municipios de la argentina que la institucionalización genera este tipo de impacto."
} 
Finalmente, la heterogeneidad de municipios bonaerenses que participan en estrategias de internacionalización, tanto en cuanto a su tamaño, ubicación interiorconurbano, como color político, dan cuenta de que se trata de una estrategianecesidad ya enraizada en las políticas públicas locales ${ }^{18}$.

\section{IV.CONCLUSIÓN}

Como hemos identificado, la realidad ha superado las restricciones normativas de los municipios. Especialmente desde la década de los 90 y en el marco de la apertura de la economía, los acuerdos de financiamiento internacionales y el desarrollo de la cooperación sur-sur y descentralizada, los municipios fueron adquiriendo mayor experiencia e iniciativa en estas políticas internacionales subnacionales.

Señalamos los principales enfoques y herramientas desde el campo de las relaciones internacionales, conceptualizando la inserción internacional de los actores subnacionales. En este marco nos adentramos a estudiar la creciente interdependencia como una de las dinámicas de la actual Sociedad Internacional Global. La interdependencia se desprende de la conceptualización de la globalización y comienza a adquirir características autónomas, hecho relevante para comprender las profundas transformaciones que experimenta la actual realidad internacional.

La visibilidad alcanzada en las últimas décadas por la política internacional subnacional permite señalar las oportunidades e identificar la complejidad del proceso que hemos reseñado. En este trabajo identificamos dos planos de análisis: en primer lugar, se analizó la creciente interdependencia como una dinámica en el marco de la actual Sociedad Internacional Global y desde esta perspectiva ubicamos la aparición de los actores de carácter subnacional, identificados en los municipios de la Provincia de Buenos Aires. Finalmente, nos detuvimos en el concepto política internacional subnacional, identificando las diversas formas de posicionamiento y relacionamientos de los municipios en el exterior deteniéndonos en ejemplos de las diversas experiencias de los municipios de la Provincia de Buenos Aires.

\footnotetext{
${ }^{18}$ Ya Calvento (2010) señalaba la tendencia a la inserción a nivel internacional de las ciudades intermedias que componen la provincia de Buenos Aires, con una alta y diversificada inserción.
} 
Sobre la base de la conceptualización realizada distinguimos un conjunto de tareas realizadas por los municipios y determinadas desde dos áreas: las de relacionamiento institucional internacional y las de posicionamiento internacional. De esta manera, se observa cómo las primeras se conforman en relación con otros actores subnacionales, mientras que las segundas, se utilizan para un posicionamiento internacional, suponiendo un mayor compromiso por parte de los gobiernos subnacionales. Por otro lado, reconocimos las diversas estrategias institucionales que llevan adelante los municipios para liderar y orientas sus políticas internacionales.

Entendemos que esta primera aproximación al tema es necesaria para destacar la creciente relevancia de esta política pública, señalando cómo los municipios se presentan como actores que buscan maximizar sus ventajas en el contexto internacional, denominado Sociedad Internacional, ahora global. Será necesario, a futuro, explorar cómo se consolidan estas políticas con un abordaje de casos que permita adentrarnos en estas nuevas relaciones internacionales.

\section{BIBLIOGRAFÍA}

Arenal Moyua, C. del. (2005). En torno al concepto de sociedad internacional. En M. Vargas Gomez-Urrutia et al. (Coords.), Soberanía del Estado y derecho Internacional. Homenaje al profesor Juan Antonio Carrillo Salcedo (pp. 453-464). Sevilla, España: Universidad de Córdoba/Universidad de Sevilla/Universidad de Málaga.

Arenal Moyua, C. del. (2008). Mundialización, Creciente Interdependencia y Globalización en las Relaciones Internacionales. En V. Gasteiz. (Ed.), Cursos de Derecho Internacional y Relaciones Internacionales (vol. 1). (pp. 181-268). España: Universidad del País Vasco/Euskal Errika Unibersitatea/Argitalpen Zerbitzua Servicio Editorial.

Batista, S., Jakobsen, K., \& Evangelista, A. C. (2008). La apertura al exterior de las ciudades latinoamericanas y la cooperación descentralizada. Montevideo, Uruguay: Observatorio de Cooperación Descentralizada Unión Europea América Latina. Recuperado de http://observ-ocd.org/sites/observ-ocd.org/files/2018-03/385 193.pdf 
Beck, U. (2008) ¿Qué es la globalización?: Falacias del globalismo, respuestas a la globalización. Buenos Aires, Argentina: Paidos. Recuperado de https://ocw.uca.es/pluginfile.php/1496/mod resource/content/1/beckulrichqueeslagloba lizacion.pdf

Bull, H. (1977). The Anarchical Society: a study of order in world politics. New York, EEUU: Columbia University Press.

Buzan, B. \& Little, R. (2000). International Systems in World History. Remaking the Study of International Relations, Oxford, UK: Oxford University Press.

Calvento, M. (2010). Estrategias de vinculación internacional en los municipios de tamaño intermedio de Argentina. Trabajo presentado en $\mathrm{V}$ Congreso Latinoamericano de Ciencia Política. Buenos Aires, Argentina. Disponible en http://www.aacademica.org/000-036/12

Calvento, M. (2012). La participación internacional de los estados subnacionales: El caso de los gobiernos municipales de tamaño intermedio de la provincia de Buenos Aires. (Tesis doctoral del Doctorado en Ciencias Políticas). UNSAM. Buenos Aires, Argentina.

Calvento, M. (2015). (Comp.). Procesos y Actores en la Gestión de la Política Internacional Subnacional. CEIPIL-ANPCyT. Tandil, Argentina. Recuperado de http://www.ceipil.org.ar/wp-content/uploads/2016/11/procesos-actores-en-la-gestionpolitica-internacional-subnacional.pdf

Calvento, M. (2016). La Política Internacional Subnacional: una propuesta para el abordaje del accionar contemporáneo en Argentina. Desafíos, 28(1). Recuperado de https://revistas.urosario.edu.co/index.php/desafios/article/view/4477/3213

Chasquetti, D. (2006). El MERCOSUR y las ciudades. Apuntes para una Agenda del Comité de Municipios del Foro Consultivo de Municipios, Estados Federados, Provincias y Departamentos del MERCOSUR. Montevideo, Uruguay: Friedrich Ebert Foundation. Recuperado de http://library.fes.de/pdf-files/bueros/uruguay/04478.pdf 
Fernández Arroyo, N. \&. Pieske, V. (Mayo 2004). Informe Especial. Cooperación Internacional para Municipios (CIPPEC). Recuperado de http://www20.iadb.org/intal/catalogo/PE/2009/02288.pdf

CONSEJO ARGENTINO PARA LAS RELACIONES INTERNACIONALES (2015). Proyecto "Sistematización y publicación on line de los Convenios Internacionales celebrados por las provincias argentinas". Recuperado de http://www.cari.org.ar/pdf/provincias-convenios-buenosaires.pdf

Di Pietro, P. (2001) Hacia un desarrollo integrador y equitativo: una introducción al desarrollo local. Recuperado de http://www.bibliotecavirtual.info/wpcontent/uploads/2011/06/hacia un desarrollo integrador y equitativo.pdf

Fronzaglia, M. L. (2005). Unidades subnacionais: um estudo de caso sobre a cidade de São Paulo - de 2001 a 2004. (Tesis de Maestría). UNICAMP. Campinas, Brasil.

Gelli, M. A. (2006). Constitución de la Nación Argentina. Comentada y Concordada. (3 Ed.). Buenos Aires, Argentina: La Ley.

Held, D., Mcgrew, A., Goldblatt, D. \& Perraton, A. (2002). Transformaciones globales. Política, economía y cultura. México: Oxford Univ. Press.

Hoffmann, S. (1990) Teorías contemporáneas sobre las relaciones internacionales. Madrid, España: Tecnos.

Keating, M. (2001). Paradiplomacia y constitución de redes regionales. Revista valenciana d'estudis autonòmics, (36), 39-50.

Keohane, R. \& Nye, J. (1988) Poder e interdependencia. La política mundial en transición. Buenos Aires, Argentina: GEL.

Maira, L. (2010). (Ed.). La Política Internacional Subnacional en América Latina. Buenos Aires, Argentina: Libros del Zorzal.

Miranda, R. (2005). Paradiplomacia y gobierno local: indicios de un modo diferente de hacer relaciones internacionales. Anuario 2005 de Relaciones Internacionales. Instituto 
de Relaciones Internacionales, UNLP. Recuperado de http://www.iri.edu.ar/publicaciones iri/anuario/CD\%20Anuario\%202005/Indices/cerpi.ht $\underline{\mathrm{m}}$

Natalizio, M. V. (2007). La Multicausalidad y Multidimensionalidad de la Acción Internacional de los Actores Subnacionales Argentinos. El caso de la Ciudad Autónoma de Buenos Aires. Buenos Aires, Argentina: CAEI.

Nye, J. S. (2003). La paradoja del poder norteamericano. Madrid, España: Taurus.

Marcio Cruz, P. \& Bodnar, Z. (Julio-Diciembre 2008). Pensar globalmente y actuar localmente: el estado transnacional ambiental en Ulrich Beck. Jurídicas, 5(2), 13-25. Recuperado de https://www.redalyc.org/pdf/1290/129012573002.pdf

Pallero, R. (Noviembre, 2014). La institucionalización de una área de relaciones internacionales en los municipios de Mendoza: ¿una deuda pendiente?. En VII Congreso del IRI, I Congreso del CoFEl y II Congreso de la FLAEI. IRI-UNLP. La Plata. Recuperado de: http://sedici.unlp.edu.ar/handle/10915/44487

Perotti, J. (Noviembre, 2010). La internacionalización de las provincias y municipios argentinos: experiencias e iniciativas del accionar externo desarrollado por las entidades subnacionales. En V Congreso de Relaciones Internacionales. Instituto de Relaciones Internacionales-Universidad Nacional de La Plata. La Plata. Recuperado de http://sedici.unlp.edu.ar/handle/10915/39740

Piana, R.S. \& Tisera, J. (2017) Globalización, interdependencia compleja y mundialización: la dialéctica entre lo global y lo local. Razón Crítica, 3, pp. 145-173, doi: http://dx.doi. org/10.21789/25007807.1240

https://papers.ssrn.com/sol3/papers.cfm?abstract id=3078211

Ruiz Alanís, L. (Enero-Abril 2009). Las relaciones internacionales de los municipios. Convergencia. Revista de Ciencias Sociales, 16(49), 253-275. Recuperado de http://www.paradiplomacia.org/upload/downloads/faf3e8a0c900cfeabe24b61b985182f0 paper-lasrelinternacdelosmunciicpios.pdf 
Truyol y Serra, A. (1993). La sociedad internacional. Madrid, España: Alianza.

Zapata Garesché, E. (2007). Manual práctico para internacionalizar la ciudad. Guía para la acción exterior de los gobiernos locales y la cooperación descentralizada Unión Europea-América Latina. (Vol. 1). Barcelona, España: Diputación de Barcelona.

Zeraoui, Z. (2008). ¿Qué es la paradiplomacia?. La Red21. Recuperado de: http://www.Ir21.com.uy/mundo/248234-que-es-la-paradiplomacia

Zubelzú, G. (2008). El diseño institucional y los perfiles de gestión externa de las provincias argentinas. En E. Iglesias, V. Iglesias \& G. Zubelzú. (Coords.), Las provincias argentinas en el escenario internacional. Desafíos y obstáculos de un sistema federal (pp. 83-106). Buenos Aires, Argentina: CARI-PNUD. Recuperado de http://www.cari.org.ar/pdf/provincias-escenario.pdf

TISERA JUAN CRUZ: Doctor En Relaciones Internacionales, Universidad del Salvador. Docente Titular Seminario de Doctorado en Metodología de las Ciencias Sociales, del Doctorado en Relaciones Internacionales y del Doctorado en Ciencia Política. Universidad del Salvador. Doctorados Acreditados CONEAU, Calificación C, Resolución No 620/99 y Resolución № 986/15. Docente Titular Seminario de Maestría: Introducción al Proyecto de Tesis. Maestría en Relaciones Internacionales. Universidad del Salvador. Maestría Acreditada ante CONEAU por Resolución 206/11. Investigador Principal Facultad de Ciencias Sociales (USAL). Instituto de Investigaciones Científicas y Sociales (IDICSO). jctiser@gmail.com

MANASSERo MARía Soledad: Magister en Relaciones Internacionales, Instituto de Relaciones Internacionales (IRI) de la Universidad Nacional de La Plata (UNLP). Licenciada en Ciencia Política y Relaciones Internacionales por la Universidad Católica de La Plata (UCALP). Doctorando en Relaciones Internacionales del Instituto de Relaciones Internacionales de la Universidad Nacional de La Plata.msmanassero@gmail.com

Piana Ricardo Sebastián: Doctor en Ciencia Jurídica, Universidad Nacional de La Plata. Doctor en Ciencia Política, Universidad del Salvador. Adjunto de la materia Derecho Político en la Facultad de Ciencias Jurídicas y Sociales de la Universidad Nacional de La Plata. r piana@yahoo.es 